\title{
Caracterização do Público-Alvo de Jogos Educacionais na área da Computação
}

\author{
Paulo E. Battistella ${ }^{1}$, Christiane G. von Wangenheim ${ }^{1}$ \\ ${ }^{1}$ Grupo de Qualidade de Software (GQS) - Instituto Nacional para Convergência Digital \\ (INCoD)-Departamento de Informática e Estatística (INE)- Universidade Federal de \\ Santa Catarina (UFSC) \\ Florianópolis - $\mathrm{SC}-$ Brasil \\ paulo@incod.ufsc.br, c.wangenheimeufsc.br
}

\begin{abstract}
Educational games can bring several benefits also for teaching computing. However, in order to obtain these benefits it is important to design these games taken into consideration the characteristics of this specific target audience. Thus, the objective of this paper is to characterize the computing students with respect to the application of educational games. Data has been collected via an online survey with the participation of 406 students. The results show that the target audience are mostly male, frequently play digital games frequently on their notebook or smartphone preferring RPG and action-adventure games. The result of our survey can help developers of games for teaching computing as part of the context analysis in game design.
\end{abstract}

Resumo. Jogos educacionais podem trazer diversos beneficios, incluindo no ensino de computação. No entanto, para obter esses benefícios é importante conceber jogos que levam em consideração a caracterização do público-alvo. Assim, o objetivo deste artigo é caracterizar os estudantes de computação com relação à aplicação de jogos educacionais. Os dados foram coletados por meio de uma pesquisa on-line com 406 participantes. Os dados mostram que o público-alvo é principalmente masculino, frequentemente jogam jogos digitais em seus notebooks ou smartphones, preferem jogos de RPG e ação-aventura. $O$ resultado da pesquisa pode ajudar os desenvolvedores de jogos educacionais, como uma parte da análise de contexto no design de jogos.

\section{Introdução}

Jogos são considerados poderosos métodos de ensino e acredita-se resultar em uma ampla gama de benefícios. Com isso, aumentando a eficácia da aprendizagem, interesse, motivação e persistência dos alunos [Kirriemuir e McFarlane, 2004; Freias, 2006; Mitchell e Savill-Smith, 2004; Prensky, 2007].

Jogos podem promover a "aprendizagem ativa" para alcançar um aprendizado profundo em um tempo de ensino aceitável e sem sobrecarga para o instrutor [Wangenheim e Shull, 2009]. Além disso, servem como meio de entretenimento para aprofundar e praticar conceitos, bem como ilustrar dinâmicas ou princípios abstratos [Hays e Singer, 1988]. Devido a suas características intrínsecas, tais como, competição, desafio e interação, podem transformar o aprendizado em uma experiência envolvente e divertida [Kafai, 2001].

Portanto, aprendizagem baseada em jogos também é uma alternativa promissora para ensinar computação no ensino superior. A utilização de jogos educacionais no 
ensino de computação está se tornando cada vez mais popular [Poulova e Klimova, 20015]. Existe uma grande diversidade de jogos para ensinar computação [Boyle et al., 2016; Battistella e Wangenheim, 2016], p.ex. para estrutura de dados (SORTIA [Battistella, Wangenheim, e Wangenheim, 2012], DEG4Trees [Barbosa et al., 2015], RPG4Sorting [Nunes e Parreira, 2015]), engenharia de software (UsabiliCity [Fereira, 2014], Simulador para Ensinar SCRUM [Gestal e Barros, 2014]) e programação (Abstração Game [Oliveira e Barros, 2013]).

Para obter os benefícios de aprendizagem esperados, os jogos precisam ser desenvolvidos de forma sistemática [Fullerton, 2008; Branch, 2009]. Seguindo abordagens de desenvolvimento de jogos educacionais (p.ex. The ELEKTRA Project [Kickmeier-Rust et al., 2006], Game-Based Learning Framework [Staalduinen e Freitas, 2011] e também ENgAGED (EducatioNAl GamEs Development) [Battistella e Wangenheim, 2015]), um dos primeiros passos é a análise do público-alvo para possibilitar o alinhamento do jogo as características, necessidades e preferência do público-alvo [Branch, 2009]. No entanto, é possível verificar que os jogos educacionais disponíveis na literatura não apresenta um levantamento sistemático do público-alvo [Battistella e Wangenheim 2016; Boyle et al. 2016].

Neste contexto, o presente artigo apresenta os resultados de uma pesquisa entre alunos de cursos de computação no ensino superior. Os resultados da pesquisa podem ajudar os desenvolvedores de jogos educacionais, como uma parte da análise de contexto no design de jogos.

\section{Definição da pesquisa}

O objetivo desta pesquisa é caracterizar o público-alvo de jogos educacionais na área de computação. Para alcançar o objetivo foi utilizado o método GQM (Goal, Question and Metric) [Basili, Caldiera e Rombach 1994]. A partir deste objetivo foram derivadas as perguntas de análise e as métricas. Para operacionalizar a coleta de dados foi projetado um questionário. A tabela 1 apresenta as questões propostas para alcançar os objetivos da pesquisa.

Tabela 1. Questões e escalas de resposta do questionário.

\begin{tabular}{|c|c|}
\hline $\begin{array}{l}\text { Caracterização dos Jogadores da Área da } \\
\text { Computação }\end{array}$ & Escala de resposta \\
\hline 1. Qual curso você está cursando ou cursou? & $\begin{array}{l}\text { Bacharelado em Ciência da Computação; } \\
\text { Bacharelado em Sistemas de Informação; } \\
\text { Bacharelado em Engenharia da Computação; } \\
\text { Bacharelado em Engenharia de Software; } \\
\text { Bacharelado em Informática; } \\
\text { Licenciatura em Computação ou Informática; } \\
\text { Tecnologia em Informática; } \\
\text { Pós-graduação em Computação. }\end{array}$ \\
\hline 2. Qual é a sua idade? & $\begin{array}{l}\text { Menor de } 15 \text { anos; Dos } 15 \text { aos } 18 \text { anos; Dos } 19 \text { aos } 22 \text { anos; } \\
\text { Dos } 23 \text { aos } 25 \text { anos; Dos } 26 \text { aos } 30 \text { anos; Dos } 31 \text { aos } 40 \text { anos; } \\
\text { Maior de } 40 \text { anos. }\end{array}$ \\
\hline 3. Qual é o seu gênero? & Masculino ou Feminino. \\
\hline 4. Qual é a sua preferência de gêneros de jogo? & $\begin{array}{l}\text { Ação-aventura; } \\
\text { RPG, Estratégia; } \\
\text { Corrida, Simulação; } \\
\text { Puzzle; } \\
\text { Quiz; } \\
\text { Adivinhação; } \\
\text { Roll-and-Move; } \\
\text { [Battistella e Wangenheim, 2015]. }\end{array}$ \\
\hline
\end{tabular}




\begin{tabular}{|c|c|}
\hline $\begin{array}{l}\text { 5. Você tipicamente prefere jogar jogos em qual } \\
\text { plataforma? }\end{array}$ & $\begin{array}{l}\text { Jogo digital de computador stand-alone p. ex. computador pessoal } \\
\text { sem acesso a internet. } \\
\text { Jogo digital de computador online p. ex. computador pessoal com } \\
\text { acesso a internet. } \\
\text { Jogo digital de console p. ex. XBox 360, Playstation, WII - } \\
\text { Nintendo. } \\
\text { Jogo digital mobile - smartphone p. ex. IPhone, Samsung Galaxy. } \\
\text { Jogo digital mobile - tablet p. ex. IPad, Samsung Galaxy Note. } \\
\text { Jogo não-digital de tabuleiro. } \\
\text { Jogo não-digital de cartas. } \\
\text { Jogo não-digital papel \& lápis (para ser jogado apenas com papel e } \\
\text { lápis.) p. ex. jogo imagem e ação, "caça-palavras". } \\
\text { Jogo não-digital acessórios (para ser jogado com acessórios ou } \\
\text { adereços (objetos portáteis)) p. ex. Lego. }\end{array}$ \\
\hline 7. Com que frequência você joga jogos digitais? & Nunca; Raramente; Mensalmente; Semanalmente; Diariamente. \\
\hline $\begin{array}{l}\text { 8. Com que frequência você joga jogos não- } \\
\text { digitais? }\end{array}$ & Nunca; Raramente; Mensalmente; Semanalmente; Diariamente. \\
\hline 9. Liste os nomes dos seus três jogos favoritos? & Campo de texto aberto. \\
\hline $\begin{array}{l}\text { 10. Quais são os seus cenários de jogos } \\
\text { favoritos? }\end{array}$ & $\begin{array}{l}\text { Esporte; } \\
\text { Medieval; } \\
\text { Dungeons\&Dragons; } \\
\text { Ficção científica; } \\
\text { Animais; } \\
\text { Outro. } \\
\text { [Battistella e Wangenheim, 2015] }\end{array}$ \\
\hline $\begin{array}{l}\text { 13. Qual sistema operacional para computador, } \\
\text { você utiliza em casa? }\end{array}$ & Windows; Linux; MacOS; outro. \\
\hline $\begin{array}{l}\text { 14. Qual é a velocidade de banda da sua } \\
\text { conexão de internet em sua casa? }\end{array}$ & $\begin{array}{l}1 \mathrm{Mb} ; 2 \mathrm{Mb} ; 4 \mathrm{Mb} ; 5 \mathrm{Mb} ; 8 \mathrm{Mb} ; 10 \mathrm{Mb} ; 20 \mathrm{Mb} ; 30 \mathrm{Mb} ; 50 \mathrm{Mb} \\
100 \mathrm{Mb} \text {; outro. }\end{array}$ \\
\hline 15. Quais navegares de internet você mais usa? & Internet Explorer; Firefox; Chrome; Safari; Opera; outro. \\
\hline $\begin{array}{l}\text { 16. Qual a resolução do monitor que você } \\
\text { utiliza? }\end{array}$ & $\begin{array}{l}176 \times 144(\mathrm{px}) ; 320 \times 200(\mathrm{px}) ; 352 \times 288(\mathrm{px}) ; 480 \times 320(\mathrm{px}) ; \\
720 \times 240(\mathrm{px}) ; 720 \times 480(\mathrm{px}) ; 768 \times 576(\mathrm{px}) ; 854 \text { x } 480(\mathrm{px}) ; \\
1920 \times 1080(\mathrm{px}) ; 2560 \times 2048(\mathrm{px}) ; 3840 \times 2400(\mathrm{px}) ; \text { outro. }\end{array}$ \\
\hline $\begin{array}{l}\text { 17. Você possui algum comentário a mais para } \\
\text { fazer sobre suas preferências de jogo? }\end{array}$ & Campo de texto aberto. \\
\hline
\end{tabular}

\section{Execução da Pesquisa}

A pesquisa foi realizada de modo online, entre novembro de 2014 até abril de 2015.0 convite de participação foi realizado virtualmente, utilizando a lista de e-mails de cursos de computação da UFSC, lista de e-mails da Sociedade Brasileira de Computação (SBC), grupos de redes sociais como Facebook e Linkedin. Ao total participaram da pesquisa 406 alunos e egressos de cursos de computação, sendo que 304 participantes responderam a pesquisa completamente.

A maior parte dos participantes são alunos ou egressos do curso de Ciência da Computação, com percentual de $40 \%$. Também participaram da pesquisa $25 \%$ dos alunos dos cursos de Sistema de Informação. A pesquisa teve a participação de $12 \%$ de alunos de cursos de pós-graduação e $8 \%$ de alunos do curso de Engenharia de Computação. Nos demais cursos, a participação não passou de 5\% dos alunos ou egressos. 


\section{Resultados}

Grande parte dos participantes (56\%) da pesquisa está entre a faixa de 19 e 30 anos de idade, o que evidencia que o público-alvo é jovem. A pesquisa evidenciou que a maioria dos alunos (84\%) é do gênero masculino.

Os jogos preferidos pelos alunos são do gênero ação-aventura e RPG, com

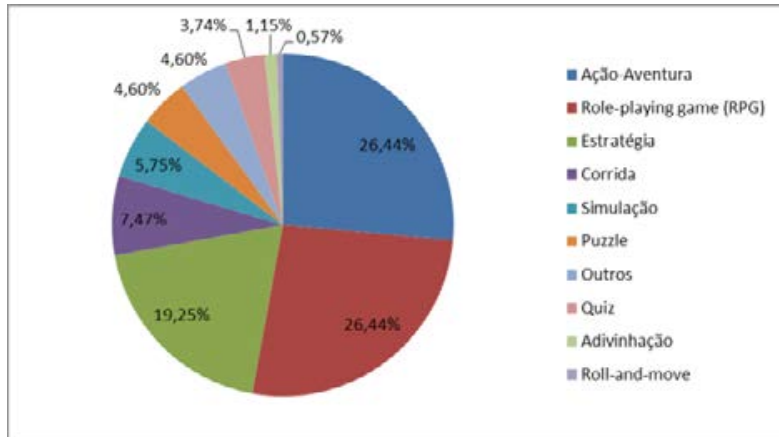

Figura 1.Preferências de gênero de jogo, entre 348 respostas de alunos. percentual de $26 \%$ para cada um dos gêneros. Os jogos do gênero estratégia apresentam $19 \%$ da preferência dos alunos. Os demais estão abaixo de $8 \%$ da preferência dos alunos. Assim, é possível inferir que $71 \%$ dos alunos preferem jogos dos gêneros Açãoaventura, RPG e estratégia juntos (figura 1).

Tipicamente os alunos preferem jogar jogos em plataformas digitais, com maior preferência nas plataformas de computador online (37\%), console (23\%), computador stand-alone (19\%) e mobilesmartphones (11\%). As demais plataformas não apresentaram mais do que 3\% da preferência dos alunos.

Considerando que entre os $93 \%$ dos alunos que preferem jogos digitais, destacase que mais de um terço dos alunos (37\%) preferem jogos online. Tipicamente estes jogos são multiplayers e do gênero RPG. Analisando as preferências dos alunos por jogos digitais, principalmente pelos jogos de computadores e console é possível inferir que o desenvolvimento de novos jogos para este público-alvo terá mais receptividade se for um jogo de computador online ou de console (Figura 2).

O modo de interação de jogos mais utilizado pelos alunos é o

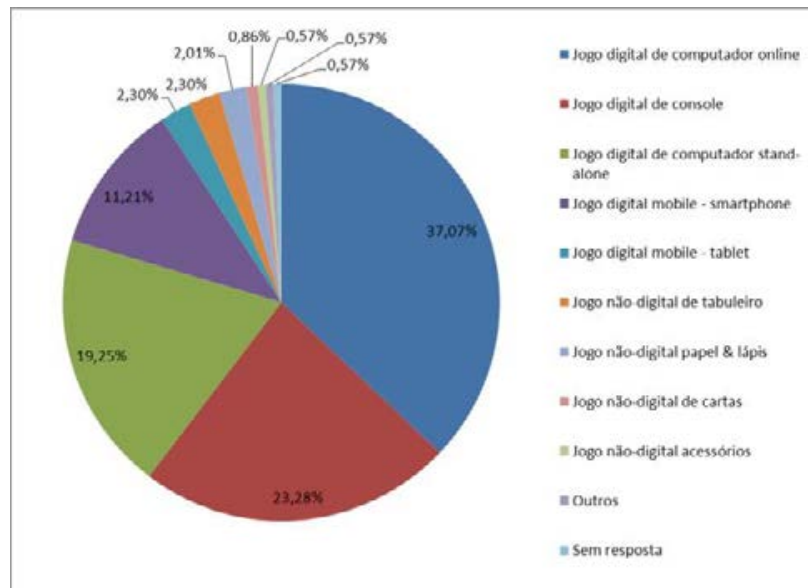

Figura 2. Plataformas de jogo de interesse entre 346 respostas de alunos.

singleplayer (41\%). No entanto, os alunos apresentam 40\% de preferência pelo modo de interação multiplayer. Ele teve $23 \%$ para multiplayers competitivos, os quais os jogadores competem entre si, e 17\% multiplayers cooperativo, os quais os jogadores colaboram entre si (figura 3 ).

Em questão de múltiplas respostas, fica evidente que os participantes preferem jogar sozinhos $(84 \%)$, normalmente em suas casas. Quando se trata de jogar com colegas, existe pouca diferença entre jogar juntos com os colegas $(40 \%)$ ou a distância (online) (47\%). Vale destacar que a opção lanhouse não houve resposta. 
A frequência dos alunos que jogam jogos digitais evidencia a preferência em jogar semanalmente (33\%), diariamente $(25 \%)$ e mensalmente $(15 \%)$. O que evidencia a preferência de $73 \%$ dos alunos em jogar jogos digitais. No entanto, $24 \%$ dos alunos raramente jogam jogos digitais, como apresenta a figura 4 .

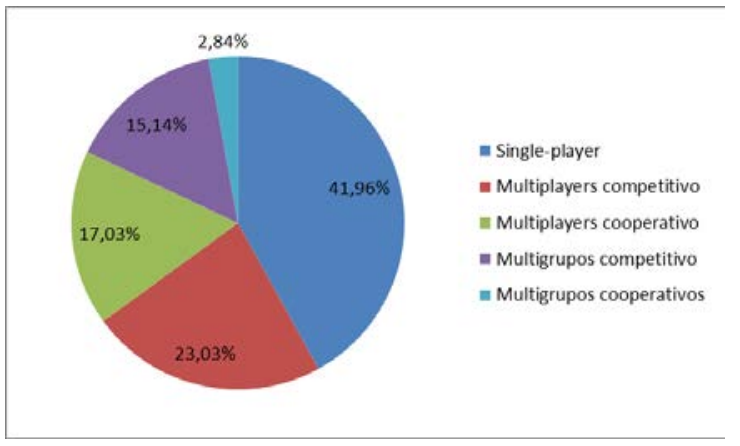

Figura 3. Modo de interação de preferência de 317alunos.

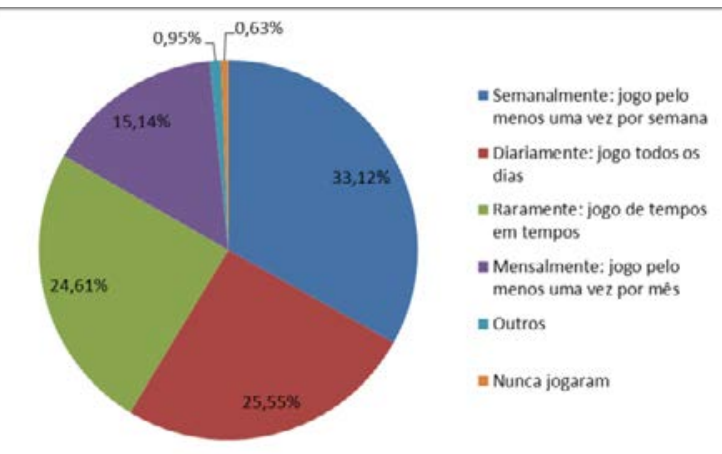

Figura 4. Frequências de jogo para jogos digitais, entre 317 respostas dos alunos.

Em relação a jogar jogos não-digitais é possível verificar que 93\% dos alunos se interessam por estes jogos. Diferentemente dos digitais, os alunos jogam raramente ou mensalmente. Isso evidencia que mesmo com muitos alunos interessados, a frequência de uso é baixa.

A frequência dos alunos que jogam jogos não-digitais é de $65 \%$ raramente, $19 \%$ mensalmente e $9 \%$ semanalmente. Apenas $4 \%$ dos alunos nunca jogam jogos nãodigitais.

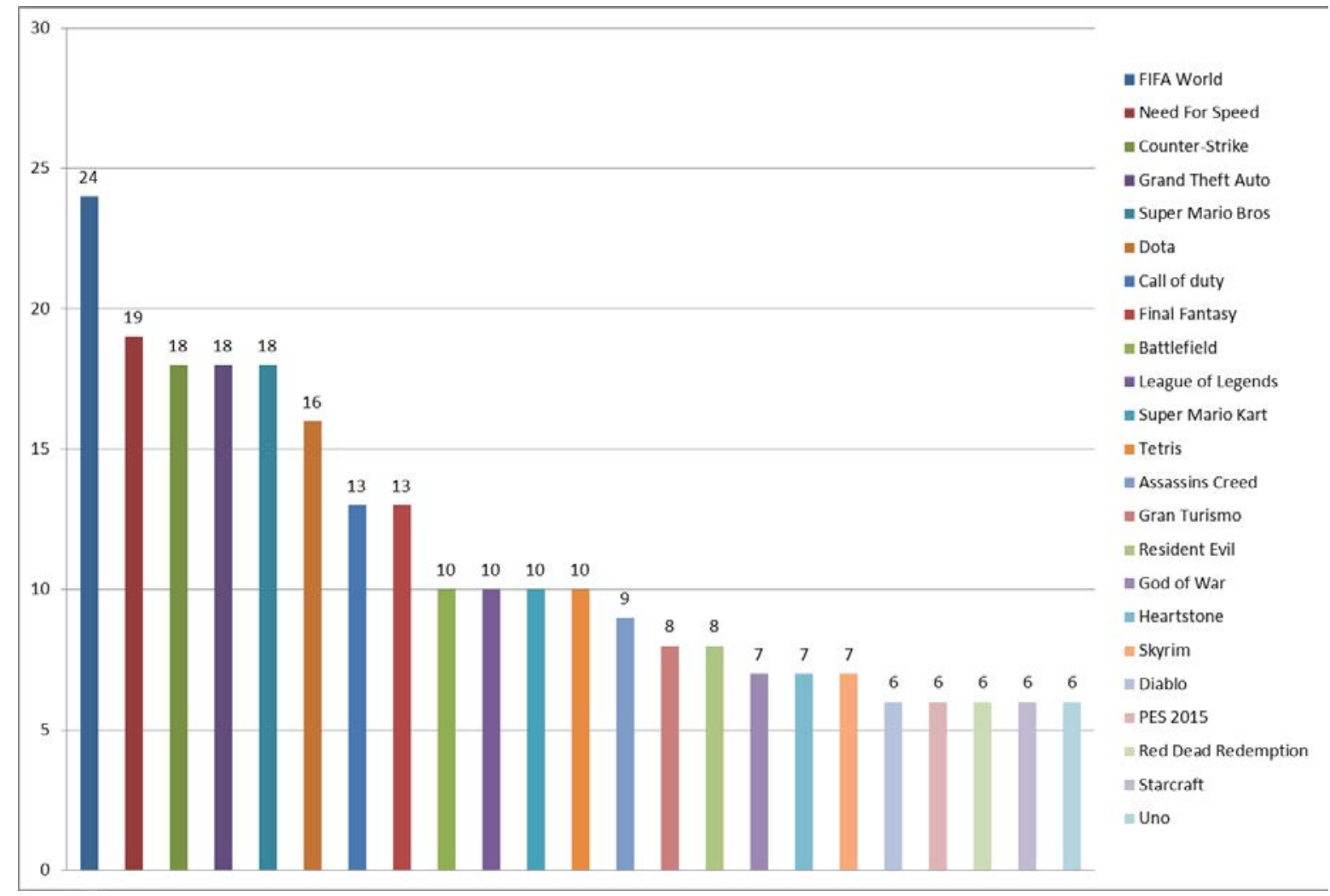

Figura 5. Ranking dos 23 jogos mais jogados, entre 310 respostas dos alunos. 
O ranking dos jogos mais jogadosapresenta234 jogos, sendo a grande maioria digital. Os 5 jogos mais citados foram FIFA World (24 citações), Need For Speed (19 citações) e Super Mario Bros, Grand Theft Auto (GTA) e Counter-strike (18 citações). Entre os 23 jogos mais citados (figura 5), destaca-se que apenas 1 é não-digital.

Os cenários preferidos pelos
alunos são medieval (51\%),
Dungeons \& Dragons $(48 \%)$ e
ficção-científica $(48 \%)$. Estes três
cenários estão ligados também a
preferência pelos gêneros de ação-
aventura e RPG. Estes cenários
tipicamente apresentam uma
narrativa interessante e que leva o
jogador à uma maior imersão ao jogo
e apresenta gráficos de qualidade
(figura 6).

$\begin{array}{ccc}\text { Em } & \text { relação } & \text { aos } \\ \text { equipamentos } & \text { eletrônicos } & \text { foi }\end{array}$ coletada mais de uma resposta por aluno. Deste modo, os jogadores optaram por mais de um equipamento utilizado para jogar. Ficou evidente que a maior parte deles são notebook, smartphone e desktop. Vale destacar que smartphone está em segundo lugar, perdendo apenas para os notebooks. Assim, fica evidente que os jogadores preferem equipamentos que permitam maior mobilidade. Por exemplo, utilizando o notebook o jogador pode jogar em vários locais, p. ex. em casa, faculdade ou reunidos em grupos de amigos (figura 7).

O sistema operacional mais utilizado para jogar é o Windows (86\%).Este fato fica evidente no Ranking dos jogos mais jogos (figura 5) por apresentar principalmente os jogos deste tipo de sistema operacional. Considerando que a questão apresenta opções de múltiplas respostas, o Linux apresentou 36\% do interesse dos alunos para jogar jogos, o MacOS 9\% e os demais sistemas operacionais 1\%.

A velocidade da conexão de banda larga foi informada por 292 alunos. Entre eles, 120 possuem internet com velocidade de $10 \mathrm{Mb}, 82$ com velocidade 1 a $5 \mathrm{Mb}$. Também 23 alunos possuem internet com velocidade de $15 \mathrm{Mb}$ e 22 participantes possuem internet entre 25 e $30 \mathrm{Mb}$. A partir destes dados, é possível inferir que menos da metade dos participantes não possuem conexão de banda larga.

Os navegadores de internet mais utilizados pelos alunos em questão de múltipla escolha evidenciou que $84 \%$ dos alunos utilizam o navegador Chrome, seguido do Firefox com 55\%. Os navegadores Safari, Opera e Internet Explorer apresentaram 
menos de $6 \%$ das preferências dos alunos. Outros navegadores apresentaram pouco mais de $1 \%$.

Entre a preferência dos alunos pela resolução de monitor fica evidente que não existe uma resolução padrão. No entanto, houve um predomínio de $22 \%$ para monitores de 22 polegadas e $19 \%$ para monitores de 17 polegadas.

Nos comentários adicionais dos alunos, apenas $15 \%$ informaram comentários sobre suas preferências de jogos. Entre essas informações é possível destacar: (a) preferência por jogos que melhorem a cognição, como Neuro Nation e Fitbrains; (b) interesse por jogos cooperativos por serem divertidos, no entanto, são raros de encontrar; (c) preferência por jogos que ofereçam liberdade para o jogador fazer o que desejar, sem ter que seguir linearmente uma sequência de passos; (d) interesse por jogos de tabuleiro, não-digitais, porém apresenta dificuldade de encontrar pessoas para jogar; (e) preferência por jogos versáteis, tendo recursos que podem ser otimizados, como no RPG.

\section{Discussão}

A partir dos dados coletados é possível concluir que atualmente o público-alvo de jogos educacionais na área da computação é na grande maioria do gênero masculino e formado por jovens. Eles têm o hábito de frequentemente jogar jogos digitais online nos seus notebooks e/ou smartphones. Em relação ao modo de interação, os alunos ficaram relativamente divididos entre singleplayer e multiplayers. Na figura 5 é possível verificar o interesse dos alunos por estes dois tipos de modo de interação, principalmente porque muitos jogos citados apresentam versões singleplayer $\mathrm{e}$ multiplayers.

Em geral eles preferem jogos de RPG e ação-aventura, preferindo cenários do tipo Dungeons \& Dragons e ficção-científica. Entre os jogos de RPG mais citados destaca-se Diablo, Dota, Final Fantasy, League of Legends, Starcraft. Entre os jogos mais citados de Ação-aventura destaca-se Super Mário Bros e Grand Theft Auto (GTA).

Com base nas respostas dos alunos, é possível verificar que grande parte deles apresentam interesse em jogos não-digitais. Porém, mesmo com grande interesse dos alunos é possível verificar que estes jogos são jogados raramente. Entre os 23 jogos listados no Ranking (figura 5), apenas um jogo é não-digital (Uno).

Em relação ao equipamento tecnológico ficou evidente a preferência dos alunos pelo Windows como sistema operacional. E também as respostas mostraram que mais da metade dos alunos possuem banda larga para acessar a internet de suas casas.

De modo geral é possível perceber um grande interesse dos alunos de computação por jogos. Deste modo, é possível utilizar este interesse para estimular o ensino de conteúdos computacionais por meio de jogos educacionais, tornando assim o aprendizado mais atrativo e divertido. Com base nesta pesquisa, o professor também pode estimular o aprendizado de gêneros ou plataformas de jogos que não são jogados com frequência, mas que são de interesse dos alunos. Por exemplo, no caso de jogos de não-digitais que podem promover cooperação ou interação entre os alunos. Ou então, a utilização de jogos educacionais que simulam uma atividade real, como nos jogos para área de Engenharia de Software Lidar com Pessoas Dificeis, SimSE e Problems and 
Programmers apresentados na revisão sistemática da literatura [Battistella e Wangenheim, 2016].

\subsection{Ameaças à validade da pesquisa}

Os resultados obtidos nesta pesquisa precisam ser interpretados com cuidado, levando em consideração as ameaças à sua validade. Entre os fatores que a ameaçam, destaca-se o impacto na validade dos resultados, principalmente pela generalização dos resultados. Outra ameaça está relacionada ao tamanho relativamente pequeno da amostra, considerando a grande quantidade de alunos de cursos de computação no Brasil. No entanto, mesmo com uma amostra relativamente pequena é possível considerar os dados analisados relevantes.

Outro fator que pode ser considerado ameaça, são as respostas dos alunos que não interpretaram as perguntas de modo adequado ou ao preencher a pesquisa esconderam informações. Outra ameaça identificada é em relação aos alunos que responderam a pesquisa, mas desconheciam o tema de pesquisa, por exemplo, no caso de alunos que não conhecem jogos, mas participaram da pesquisa.

Outra ameaça à validade é o fato de poucos alunos de cursos como Bacharelado em Informática, Licenciatura em Computação ou Informática e Bacharelado em Engenharia de Software terem participado da pesquisa, mostrando que a caracterização do público-alvo realizada está voltada principalmente para os alunos dos cursos de Ciência da Computação e Sistema de Informação.

Outra ameaça identificada é em relação aos gêneros de jogos educacionais. Atualmente os gêneros não apresentam uma taxonomia padrão que seja utilizada amplamente na literatura. Muitas vezes estas taxonomias apresentam os jogos educacionais como um gênero, assim como ação-aventura, estratégia, puzzle, o que torna difícil a caracterização deste gênero. Para minimizar esta ameaça, foi utilizada a taxonomia criada para o processo ENgAGED [Battistella e Wangenheim 2015], na qual foi criada uma taxonomia de gêneros de jogos (digitais e não-digitais) para classificar os jogos educacionais desenvolvidos para área da computação com base nos gêneros disponíveis na literatura e sites de jogos online.

\section{Conclusão}

$\mathrm{O}$ artigo apresentou uma pesquisa para caracterizar os perfis de jogadores da área da computação. Participaram da pesquisa 406 alunos e egressos dos cursos de computação, porém, apenas 304 alunos responderam completamente. Os resultados evidenciam que a maioria dos alunos são do gênero masculino e jovens entre 19 e 30 anos de idade.

A preferência por jogos mostra um predomínio dos interesses dos alunos por jogos online e dos gêneros Ação-aventura e RPG. O ranking com os 23 jogos mais citados também evidencia esta preferência. Além disso, a preferência também mostra que os jogadores jogam com grande frequência os jogos digitais e com pouca frequência os não-digitais.

Em relação a preferência por equipamentos eletrônicos, a pesquisa mostra que o Windows é o sistema operacional mais utilizado. E também, mais da metade dos alunos possuem acesso a conexão de banda larga e utilizam com frequência seus notebooks e 
smartphones para jogar. Assim, fica evidente o interesse dos alunos por jogos online que possam ser jogados por equipamentos que permitam mobilidade.

A pesquisa realizada neste artigo permitiu a caracterização dos perfis de jogadores, fase essencial do processo de Design Instrucional e Design de Jogos. Deste modo, os resultados apresentados podem ser aplicados no desenvolvimento de novos jogos educacionais para área de computação.

\section{Agradecimentos}

Este trabalho foi apoiado pelo $\mathrm{CNPq}$ (Conselho Nacional de Desenvolvimento Científico e Tecnológico), uma entidade do governo brasileiro focada no desenvolvimento científico e tecnológico e pela CAPES (Coordenação de Aperfeiçoamento de Pessoal de Nível Superior).

\section{Referências}

Barbosa, W. A. et al. (2015) "DEG4Trees: Um Jogo Educacional Digital de Apoio ao Ensino de Estruturas de Dados", Workshop sobre Educação em Computação, Recife, Pernambuco, Brasil.

Basili, V., Caldiera, G. e Rombach, H. D. (1994) “Goal Question Metric Approach", Encyclopedia of Software Engineering, John Wiley \& Sons, Inc., p. 528-532.

Battistella, P. E., Wangenheim, A. e Wangenheim, C. G. von. (2012) "SORTIA - Um Jogo para Ensino de Algoritmo de Ordenação: Estudo de caso na Disciplina de Estrutura de Dados", Simpósio Brasileiro de Informática na Educação, Rio de Janeiro, RJ, Brasil.

Battistella, P. E. e Wangenheim, C. G. von. (2016) "Games for Teaching Computing in Higher Education - A Systematic Review", IEEE Technology and Engineering Education.

Battistella, P. E. e Wangenheim, C. G. von, (2015) "ENgAGED: Processo de Desenvolvimento de Jogos para Ensino em Computação", Relatório Técnico INCoD/GQS.01.2015.P, Florianópolis, Brasil.

Boyle, E. A. et al. (2016) "An update to the systematic literature review of empirical evidence of the impacts and outcomes of computer games and serious games", Computers \& Education, 94, pp. 178-192.

Branch, R. M. (2009) "Instructional Design: The ADDIE Approach", Springer, New York, EUA, p. 203.

Ferreira, B. et al. (2014) "UsabiliCity: Um Jogo de Apoio ao Ensino de Propriedades de Usabilidade de Software Através de Analogias", Simpósio Brasileiro de Informática na Educação, Dourados, MS, Brasil.

Freitas, S. I. de (2006) "Using games and simulations for supporting learning", Learning, Media and Technology, Digital Games and Learning, 31(4), p. 343-358.

Fullerton, T. (2008) "Game design workshop: A playcentric approach to creating innovatives games”, $2^{\mathrm{a}}$ ed., Elsevier. 
Gestal, P. R. E. e Barros, R. M. de. (2014) "Proposta de Um Simulador para Auxiliar no Processo de Ensino do Scrum", Simpósio Brasileiro de Sistemas de Informação, Londrina, PR, Brasil.

Hays, R. T. e Singer, M. J. (1988) "Simulation fidelity in training system design: Bridging the gap between reality and training", Recent Research in Psychology, Springer, p. 415.

Kafai, Y. B. (2001) "The educational potential of electronic games: From games-toteach to games-to-learn", Conference on playing by the rules: the cultural policy challenges of video games. Chicago, IL, EUA.

Kickmeier-Rust, M. D. et al. (2006). "The ELEKTRA project: Towards a new learning experience", In M. Pohl, A. Holzinger, R. Motschnig, \& C. Swertz: M3 Interdisciplinary Aspects on Digital Media \& Education, Vienna, p. 19-48.

Kirriemuir, J. e McFarlane, C. A. (2004) "Literature Review in Games and Learning Literature Review in Games and Learning”, Bristol: FutureLab Series.

Mitchell, A. e Savill-Smith, C. (2004) "The use of computer and video games for learning: a review of the literature", Learning and Skills Development Agency, Ultralab, London, UK.

Nunes, I. de F. e Parreira Junior, P. A. (2015) "RPG4Sorting - Um Jogo Educacional para Auxílio ao Ensino de Métodos de Ordenação", Workshop sobre Educação em Computação, Recife, Pernambuco, Brasil.

Oliveira, E. D. de. e Barros, R. M. de. (2013) “ABstração Game: Um Jogo Para Facilitar o Processo de Ensino e Aprendizagem da Orientação a Objetos", Workshop sobre Educação em Computação, Maceió, AL, Brasil.

Poulova, P. e Klimova, B. (2015) "Education in Computational Sciences", International Conference on Computational Science. Reykjavík, Iceland, p. 1996-2005.

Prensky, M. (2007) “Digital game-based learning”, Paragon House, p.464.

Staalduinen, J. v. e Freitas, S. de. (2011) "A Game-Based Learning Framework: Linking Game Design and Learning Outcomes”, Nova York: Peter Lang, p. 29-45.

Wangenheim, C. G. von e Shull, F. (2009) “To game or not to game?”, IEEE Software, 26(2), p. 92-94. 